\title{
Validity of predictive equations for estimating resting metabolic rate in women during the different phases of the menstrual cycle
}

\author{
M. Campolier, S. Thondre, M. Clegg and H. Lightowler \\ Functional Food Centre, Department of Sport and Health Sciences, Oxford Brookes University, Gipsy Lane, \\ Headington, Oxford, $O X 3 O B P$
}

The assessment of resting metabolic rate (RMR) becomes fundamental when determining energy requirements of individuals. Due to the increased cost of measuring RMR, predictive equations from different populations were formulated to estimate RMR. However, studies have found a lack of agreement between measured (RMRm) and predicted RMR (RMRp) ${ }^{(1)}$. Moreover, the effect of the menstrual cycle (MC) on the accuracy of these predictions has never been studied before. Some studies have shown that RMRm fluctuates within a $\mathrm{MC}^{(2,3)}$.

Eleven healthy women, age 26.6 (SD 5.9) y, BMI 22.7 (SD 2.2) kg/m² with regular MC (25-35 days), were tested three times a week during a MC. Subjects attended the laboratory after an overnight fast for the assessment of their RMR and ovarian hormone levels. RMR was measured for $30 \mathrm{~min}$ by indirect calorimetry using a ventilated hood system. Plasma ovarian hormones were analysed by an Electrochemiluminescense Immuno-Assay. Averaged RMRm of the entire MC and per phase were compared to RMRp from ten different equations valid for adults ${ }^{(4)}$. Bias was determined as the mean percentage difference between RMRm and RMRp and accuracy as the percentage of participants with an RMRp within $\pm 10 \%$ of RMRm.

\begin{tabular}{|c|c|c|c|c|c|c|c|c|}
\hline & \multicolumn{2}{|l|}{ MC } & \multicolumn{2}{|l|}{ MPh } & \multicolumn{2}{|l|}{ FPh } & \multicolumn{2}{|l|}{$\mathbf{L P h}$} \\
\hline & $\overline{\text { Bias (\%) }}$ & Accurate $(\%)$ & $\overline{\text { Bias (\%) }}$ & Accurate $(\%)$ & $\overline{\operatorname{Bias}(\%)}$ & Accurate $(\%)$ & $\overline{\text { Bias (\%) }}$ & Accurate $(\%)$ \\
\hline Harris Benedict & 13 & 45 & 12 & 55 & 10 & 36 & 15 & 36 \\
\hline Scholdfield_1 & 16 & 45 & 16 & 45 & 13 & 45 & 19 & 27 \\
\hline Scholdfield_2 & 16 & 36 & 15 & 45 & 13 & 45 & 19 & 27 \\
\hline Mifflin & 18 & 36 & 17 & 27 & 15 & 45 & 20 & 18 \\
\hline Muller_1 & 17 & 45 & 17 & 36 & 14 & 45 & 20 & 36 \\
\hline Muller_2 & 16 & 45 & 15 & 45 & 13 & 45 & 18 & 36 \\
\hline Muller_3 & 18 & 45 & 17 & 36 & 15 & 45 & 21 & 27 \\
\hline Muller_4 & 16 & 45 & 16 & 45 & 13 & 36 & 19 & 36 \\
\hline Henry_1 & 19 & 36 & 19 & 36 & 16 & 45 & 22 & 27 \\
\hline Henry_2 & 19 & 36 & 18 & 27 & 16 & 36 & 22 & 18 \\
\hline
\end{tabular}

Values are the mean bias and accurate predictions (\%) of the different predictive equations from $\mathrm{RMRm}$ as an average of $\mathrm{MC}$ and the $\mathrm{MPh}, \mathrm{FPh}$ and $\mathrm{LPh}$ (menstrual, follicular and luteal phases, respectively).

RMRp underestimated RMRm (1638 (SE 82 ) kcal) by $~ 17 \%$ in all predictive equations with $<50 \%$ of the women having an accurate RMRp. Moreover, the predictive error was magnified in the $\mathrm{LPh}$ as the bias and the prevalence of inaccurate predictions increased. Nevertheless, RMRm did not rise significantly in the luteal phase ( $\mathrm{LPh})$ compared to the menstrual (MPh) and follicular $(\mathrm{FPh})$ phases, 1674 (SE 82) vs. 1627 (SE 93), 1593 (SE 73) kcal/d, respectively $(\mathrm{p}=0 \cdot 178)$.

To conclude, the selected predictive equations might not be valid in this population and they are more biased in the LPh of the MC.

1. Weijs PJM \& Vansant GAAM (2010). Clin. Nutr. 29, 347-51.

2. Solomon S, Kurzer M \& Calloway D (1982). Am. J. Clin. Nutr. 36, 611-616.

3. Howe JC, Rumpler W V. \& Seale JL (1993). J. Nutr. Biochem. 4, 268-273.

4. European Food Safety Authority (EFSA) (2013). EFSA J. 11, 1-112. 